\title{
BIOFLAVONOIDS WITH ANTICANCER ACTIVITY AND THEIR NOVEL FORMULATIONS
}

\author{
PADMAVATHY J, SATHESH KUMAR S*
}

Department of Pharmaceutics, School of Pharmaceutical Sciences, Vels Institute of science, Technology and Advanced Sciences (VISTAS), Chennai, Tamil Nadu, India. Email: sathesh2000@gmail.com

Received: 10 October 2018, Revised and Accepted: 11 December 2018

\section{ABSTRACT}

Context: Nature blesses human with a lot of natural products with a wide range of medicinal properties from plants, animals, marine animals, and microorganisms. Among these natural sources, plant origin drugs constitute around $25 \%$ which includes various secondary metabolites such as alkaloids, bioflavonoids, terpenes, saponins, glucosides, and lignans. The bioflavonoids belonging to the polyphenol group possess various therapeutic activities such as antioxidant, hepatoprotective, antibacterial, anti-inflammatory, anticancer, and antiviral.

Objectives: The main objective of this article is to collectively present the research data published worldwide about the anticancer activity of bioflavonoids by loading them in novel formulations. Thus, the present review explored the novel formulations of the bioflavonoids with improved pharmacokinetic properties along with the enhanced anticancer activity.

Methods: A systematic scientific review was made across the peer-reviewed scientific journals and books to collect the data pertaining to areas research related to the application of bioflavonoids for treatment of cancer using novel pharmaceutical formulations.

Results: The major drawback with bioflavonoids is its poor solubility and bioavailability, which restricts the usage of bioflavonoids in the treatment of cancer in the market worldwide. Novel drug delivery system seems to possess many benefits like site-specific drug delivery along with minimal side effects and improving its pharmaceutical and therapeutic properties of drugs compared to a conventional dosage form of bioflavonoids.

Conclusion: The scope for improvement of anticancer activity of bioflavonoids by incorporating in novel pharmaceutical formulations like nanoparticles is very high, and it has to be considered as a potential area of research.

Keywords: Bioavailability, Bioflavonoids, Natural product, Novel formulations, Therapeutic properties.

(C) 2018 The Authors. Published by Innovare Academic Sciences Pvt Ltd. This is an open access article under the CC BY license (http://creativecommons. org/licenses/by/4. 0/) DOI: http://dx.doi.org/10.22159/ajpcr.2018.v11s4.31731

\section{INTRODUCTION}

Mother Nature blessed the humans with a lot of sources with valuable therapeutic properties which serves as a root for various modern era drugs in all traditional systems of medicine as well as an allopathic system of medicine. Greeneries, animal, microbes, and minerals form the sources of drugs from our environment [1]. The natural products obtained from the greeneries are usually the secondary metabolites that are not highly involved in the primary stages of growth and development, but they play an important role in the defense mechanism of the plant thereby increasing the lifespan of the plants [2]. A wide range of secondary metabolites produced by the plants is found to possess therapeutic activity in humans also. Many of the secondary metabolites from the greeneries have been used as lead molecules for the development of many of the synthetic drug products for the treatment of chronic diseases such as cancer and brain disorders. A few to quote are alkaloids, bioflavonoids, terpenes, saponins, glucosides, and lignans, etc. Campothecin, paclitaxel, vincristine, vinblastine, digoxin, nicotine, ephedrine, lobeline, and emetine are few constituents derived from plants which have found a long way in the pharmaceutical industry in fabrication as drug product [3].

Bioflavonoids, belonging to the polyphenolic compounds, are found to be widely distributed in vegetables, cereals, and fruits forming a part of human diet. Many subclasses of flavonoids are identified which includes flavones, flavonols, flavanones, isoflavones, catechins, anthocyanins, dihydroflavonols, and chalcones [4-7] and are listed in Table 1 along with their food sources. Around 3000 bioflavonoids have been identified for their useful properties. In the recent years, there is a upswing interest in the beneficial efficiency of the polyphenolic compound especially the bioflavonoids [8]. The bioflavonoids are found to possess proven multifaceted therapeutic properties such as anti-atherosclerotic effect [9], anti-inflammatory effect [10-13], anti-thrombogenic effect, antitumor effect [14-16], antiosteoporotic effect [17], hepatoprotective [18], and antimicrobial effect [19-21]. Bioflavonoids are proved to possess antioxidant property, hinders lipid peroxidation [4], and free radical scavenging property to a larger extent [22].

Cancer, a deadly disease of this era is found to have a multifaceted etiology which involves chemical, physical, environment, metabolic, and genetic factors, which forms direct or indirect cause for the disease and has been an area of recent concern for the development of the drugs for its effective treatment [23]. The rate of occurrence of cancer is found to be growing at an alarming rate and is predicted to have an occurrence of $>15$ million by the year 2020 [24,25]. Various treatment options are available for the treatment of cancer which includes, hormonal therapy, chemotherapy, surgery, and radiation therapy but the major drawbacks with the available treatment is its unwanted effects after its usage which provokes an alternative mode of treatment of cancer involving the natural products which are found to be safe and equally effective for the treatment of cancer at every stage.

Around $60 \%$ of the anticancer drugs are found to be of natural origin, and they are synthetically derived as drugs for the treatment of various cancers such as vincristine, vinblastine, actinomycin, taxoids, and anthracyclines [26]. Among the natural compounds, the flavonoids are known to possess many of the valuable pharmacological properties. The antioxidant property of bioflavonoids contributes to various other therapeutic activities such as anticancer, anti-inflammatory, antidiabetic, antiatherosclerotic, and antimutagenic. The major 
Table 1: Subclasses of flavonoids with their dietary sources

\begin{tabular}{llll}
\hline S. No. & Subclass & Dietary sources & Examples of bioflavonoids \\
\hline 1. & Flavonols & Apple, tomato, cherry, tea, garbanzo beans, onions, and red wine & Myricetin, kaempferol, quercetin, rutin \\
2. & Flavones & Celery, bell peppers, parsley, apples, and thyme & Chrysin, luteolin, and apigenin \\
3. & Flavanols & Bananas, blueberries, peaches, tea, pears, and apple & Gallocatechin and catechin \\
4. & Flavanones & Grapes and orange & Hesperidin, eriodictyol, and naringenin \\
5. & Isoflavones & Soybean and legumes & Daidzein, glycetin, genistein, and formononetin \\
6. & Anthocyanidins & Oranges, olives, red onion, sweet potato, kidney beans, black beans & Cyanidins, pelargonidin, peonidin, petunidin \\
\hline
\end{tabular}

mechanisms by which the bioflavonoids are shown to exhibit anticancer activity are by blocking the cell cycle, hindering angiogenesis and by urging apoptosis by which the further propagation of the cancer cells is ceased [27].

Many epidemiological studies have proved the anticancer potential of various subclasses of bioflavonoids. A few to quote are polymethoxyflavones are proved to hinder the growth of human leukemic cell lines (HL 60) [28], nobiletin hindered the mushrooming and relocation of human umbilical endothelial cells of human prostate, breast, skin and colon carcinoma cell lines [29], luteolin -7 methyl ether exhibited the celltoxicity towards the human lung cancer cell lines [30], genistein, genistin, daidzein, biochanin A belonging to isoflavonones subclass of bioflavonoids are found to hinder the growth of human bladder and murine camcer cell lines [31], quercetin was found to be useful in the treatment of gastric, pancreatic and thyroid cancer [32].

There are many number of studies which have investigated and proved the anticancer potential of various subclasses of bioflavonoids that have put them under the scientific area of research hoping to have a better treatment regimen for the treatment of one of the deadly disease like cancer. This has paved the way for the development of the novel formulations of bioflavonoids to overcome the conventional problems faced with it in formulating.

Novel drug delivery system development, a multifaceted area in the drug development which involves the modish design in regulating the pharmacodynamics, pharmacokinetics, immunogenicity, and nonspecific toxicity of the newly invented drugs [33]. This system alters the properties of the drugs by the usage of various polymers of natural or synthetic origin, biodegradable type, stimuli-sensitive type and even targeting of the drug to the specific organ can be achieved by various novel techniques, which have increased the burden on the scientists for deeper research in these areas. The novel drug delivery system shows many benefits compared to the conventional dosage forms, such as high bioavailability, improved remedy for chronic diseases, good patient compliance, lesser toxicity to the organs due to targeting, and so on which urges the drugs to be developed as novel formulations such as nanoparticles, liposomes, nanospheres, microspheres, hydrogels, ethosomes, and so on.

Novel techniques are introduced for formulating the natural products which are gaining the utmost importance in the field of medicine for the treatment of diseases such as cancer, brain disorders, and so on. Many bioflavonoids were evaluated for the anticancer activity and were proved to be much effective in the treatment of the same. A few of them with proved anticancer activity are fisetin, genistein, diadzein, quercetin, diosmetin, and so on [34]. Although bioflavonoids are proved to have pharmacological activity against the cancer cells, their usage is limited due to its various pharmacokinetic properties such as low solubility, less bioavailability which urges the pharmacy scientist to convert this much useful natural product into a novel formulation. This is considered to be the latest area of research in the pharmaceutics.

The flavonoids are found too much useful compounds for treating cancer by various mechanisms such as targeting apoptosis signal and inducing the process, inhibition of epidermal growth factor receptor and extracellular signal-regulated kinases pathway, modulating the Raf/MEK/ERK signaling pathway, reducing the notch signaling pathway, inhibition of Wnt/ $\beta$-catenin pathway, inhibiting the transcription factor signal transducer and activator of transcription 3 , triggering the Nf- $\square \mathrm{B}$ and Nrf2 signals, and so on [25].

This review aims in bringing into limelight the bioflavonoids possessing the anticancer potential that is formulated into the novel formulations such as nanoparticles, nanospheres, liposomes, microspheres, hydrogels, ethosomes, nanoemulsions, and so on which will surely create a revolution in the field of medicine in curing various human ailments.

\section{BIOFLAVONOIDS WITH ANTICANCER ACTIVITY AND THEIR NOVEL FORMULATIONS}

\section{Quercetin}

Quercetin, a dietary flavonoid is considered to be the pillar for many of the other flavonoids. It is found to be distributed naturally in many of the diet foods such as tea, berries, apple, onion, vegetables and nuts, seeds, barks, leaves, and flowers. It is chemically $3,5,7,3$ ', 4' pentahydroxy flavones and it is found to show many pharmacological properties such as cardioprotective effect [35], antioxidant activity, analgesic activity, antiviral activity, antidiabetic activity, anticancer activity, immunosuppressor activity, antithyroid activity, and so on. The anticancer activity was shown to be exhibited by various mechanisms such as abolishing the free radicals, reaction with the estrogen receptors, proteins and transcription factors, retarding cell proliferation, initiation of apoptosis, and reducing metalloproteinase 2 and 9 expression. Quercetin, although, has many of the therapeutic properties its use is limited due to many of the drawbacks such as less bioavailability and poor aqueous solubility and so to improve its physicochemical properties and also to improve its targeting property the novel drug delivery systems such as nanoparticles, niosomes, and liposomes can be developed for quercetin.

Saha et al. formulated and evaluated the nanoparticles of polylactic coglycolic acid with hydrophobic anticancer drugs of anthracycline origin such as paclitaxel, doxorubicin, and bioflavonoid quercetin, a chemoprevention by single emulsion solvent evaporation method. The surface modification of the bioflavonoid nanoparticles was carried out using the polymers of biological origin such as histones or bovine serum albumin. The formulated nanoparticles were evaluated for their physicochemical properties such as particle size analysis, zeta potential, entrapment efficiency, drug loading capacity, Fourier-transform infrared, differential scanning calorimetry, thermogravimetric analysis, scanning electron microscope, transmission electron microscopy, and in vitro drug release and the effects were found to be satisfactory. The main problem associated with the anticancer drugs is the opsonization which leads to the clearance of the drug by liver and spleen which was overcome by the surface modification which helps the targeting of the drug to cancer cells and for overcoming the multidrug resistance [36].

Devendiran et al. green synthesized gold nanoparticles containing the drug doxorubicin and the antioxidant bioflavonoid quercetin as a reducing agent with dextran sulfate as a stabilizing agent, adorned with folic acid and evaluated for the treatment of breast cancer. They were evaluated for their in vitro physicochemical properties and biological properties and were found to be stable. The anticancer activity was carried out using the human breast cell lines MCF-7 using MTT assay, and it was proved that the formulated nanoparticles represented a potential targeted drug delivery for better treatment of cancer [37]. 
Pandey et al. synthesized nanoparticles of quercetin embedded with polylactic acid by novel emulsified nanoprecipitation method and evaluated for its anticancer activity in human breast cancer cell lines. The physicochemical properties evaluation showed that the flavonoid release from the polymer matrix showed a sustained release and in vitro cytotoxicity studies proved the efficiency of the nanoformulation for treating cancer [38].

Sahu et al. formulated the quercetin nanoparticles of biocompatible nature using the biocompatible and nonbiodegradable polymers such as ethyl cellulose and Tween 80 by the nanoprecipitation technique for the topical prolonged delivery of the bioflavonoid for the treatment of skin cancer. The formulated nanoparticles were evaluated for their physicochemical and ex vivo skin penetration studies. From the studies, it was concluded that the quercetin formulated as nanoparticles can be sustained in the drug release thereby intending the decrease in the frequency and dose of the drug urging the research of quercetin novel formulations in the area of anticancer study [39].

\section{Fisetin}

Fisetin, known popularly as natural brown [40], is chemically 3,3', 4',7-tetrahydroxy flavone and a dietary flavonoid found in the foods such as tomatoes, onions, mangoes, apples, strawberries, cucumbers, kiwis, nuts, and grapes [40]. Fisetin was proved to possess many therapeutic activities such as antioxidant activity [41], antiinflammatory activity [42,43], antidiabetic activity [40], antiviral activity $[44,45]$, cardioprotective effect, and anticancer activity [46,47]. Fisetin bioflavonoid was found to possess much effective therapeutic activity against cancer through various mechanisms such as inducing apoptosis of cancer cells, activation of 5' Adenosine monophosphateactivated protein kinase and acetyl coenzyme A carboxylase [48-50] and so on. Since, cancer is the dreadful disease which has gained specific attention of the researchers and fisetin a natural bioflavonoid in novel formulation possesses many advantages of enhanced pharmacokinetic properties, safety, economic, and specific organ targeting which can greatly help the physicians to treat cancer in a better means.

Ragelle et al. formulated fisetin a well proved antitumor agent to improve its aqueous solubility into an nanoemulsion formulation and proved its improvement in pharmacokinetic property and its antitumor therapeutic activity on intraperitoneal administration of the fisetin nanoemulsion in Lewis lung carcinoma affected mice [51].

Bothiraja et al. formulated fisetin a flavonoid with poor water solubility and the one that undergoes more metabolism in vivo, as a nanocochelates, by trapping technique in which the flavonoid loaded dimyristoyl-phosphatidyl choline loaded liposomal vesicles were converted into the nanochelates in the presence of $\mathrm{Ca}^{2+}$ ions. This nanochelates were proved to be much stable and more effective in the treatment of human breast cancer [52].

Ghosh et al. nanoparticles of fisetin using human serum albumin by desolvation technique and evaluated for their physicochemical properties and cytotoxic activity. The formulated albumin nanoparticles were found to possess antioxidant activity and also effective against the breast cancer MCF-7 cell lines. The nanoparticles were found to be much effective in targeting the flavonoid fisetin to the cancer cells [53].

\section{Genistein}

Genistein, a nutraceutical [54] soy isoflavone and a plant estrogen, a flavanoid found in soybeans and soy food is found to posesses a variety of pharmacological activities including the anticholesterol activity, antioxidant activity, antidiabetic activity, menopausal syndrome, and anticancer activity [55]. Although genistein is a proved isoflavone for the treatment of cervical, breast and prostrate cancer, its use in this area is limited due its solubility and bioavailability, which initiates its formulation development as nanoformulation which can overcome this limitation and prove its potentiality in the treatment of cancer.
Zhang et al. produced flavonoid, genistein d- $\alpha$-tocopheryl polyethylene glycol 1000 succinate b polycaprolactone nanoparticles by modified nanoprecipitation method. This novel formulation of the flavonoid was found to be effective in improving the solubility characteristics of the antitumor flavonoid genistein and as a valuable antitumor agent in treating cervical cancer as tested in the HeLa xenograft tumor model in BALB/c nude mice [56].

Phan et al. formulated the novel liposomal formulation containing the most proven flavonoid with antitumor potential of genistein for proving its efficacy in breast, ovarian, and prostate cancers. Genistein conventional and stealth liposomes were formulated using the phospholipids and cholesterol, and its antioxidant efficacy retention was proved by the neutralization of peroxides and microscopic fluorescent probe oxidation assays. The anticancer efficacy of the genistein loaded liposomal formulation was proved in human and mouse cancer cell lines which was indicated by the measurement of specific proapoptotic activity [57].

Patel et al. studied the novel formulation of isoflavone genistein using the polymers in varying ratios of chitosan and Eudragit and evaluated them for their physicochemical properties which proved them to be valuable drugs for the treatment of dreadful disease cancer [58].

Cai et al. formulated and evaluated the chitosan linked and folic acid linked nanoparticle containing bioflavonoid genistein for its improved activity in cervical cancers. It was proved from the study that the tagging of folic acid to the genistein nanoparticles improved its cytotoxicity studies as proved by MTT assay as compared to the genistein loaded nanoparticles in the treatment of cervical cancers [59].

Aditya et al. showed the efficacy of coloading of curcumin and genistein in nanostructured lipid carriers in the treatment of antiprostrate cancer. It was proved from the studies that coloading improved the flavonoidal loading efficiency as well as the cancer cell growth inhibition property of the flavonoids to a greater extent [60].

\section{Isorhamnetin}

Isorhamnetin, a metabolite of quercetin, which is chemically 0 -methylated flavonol found in plenty in blackberries, cherries, pears, and apples [61]. The flavonoid is a highly active molecule showing wide variety of pharmacological activities such as antidiabetic, antioxidant, cardiovascular, anticancer, anti-inflammatory, hepatoprotective, and anti-obesity agent [61]. Isorhamnetin was proved to be an effective anticancer agent for treating lung cancers [62-64]. However, its targeting capacity is poor due to the poor aqueous solubility and bioavailability, which proves the necessity for the development of the nanoformulations for the most eligible flavonoid molecule in the treatment of cancer.

Settu and Vaiyapuri studied the effect of poly lactic-co-glycolic acid isorhamnetin nanoparticles on HepG2 cancer cell lines and concluded that the nanoformulation of isorhamnetin produced a significant increase in the inhibition of proliferation as compared to the plain isorhamnetin [65].

\section{Kaempferol}

Kaempferol, a plant nutrient found widely distributed in many of the plant families, such as Aspleniaceae, Aspidiaceae, Polypodiaceae, Blechnaceae, Cyatheaceae, Equisetaceae, and Ophioglossaceae and is present as a major constituent inedible foods such as onion, mustard, turnip greens, tea, grapefruit, tea, cucumber, fennel, and peach $[66,67]$. Kaempferol based plant food intake reduces the risk of various types of cancers such as lung cancer, pancreatic cancer, ovarian cancer, and gastric cancer and many of the cardiovascular disease. From various epidemiological studies, kaempferol was also proved to possess antioxidant activity, anti-inflammatory activity, antidiabetic activity [68], and antimicrobial activity [69]. Although there are many pharmacological activities; their usefulness is limited due to less oral 
bioavailability and low water solubility. Hence, researchers are carried out to improve its pharmacokinetic properties, in turn, the therapeutic benefits of the flavonoid be made completely available to the suffering patients.

Luo et al. studied the comparative effect of the most proven bioflavonoid kaempferol formulated as nanoparticle on the ovarian cancer cells and normal cells. The non-ionic polymers poly(ethylene oxide)-poly(propylene oxide)-poly(ethylene oxide) and poly (DL- Lactic acid-co-glycolic acid) were used to formulate the nanoparticles containing kaempferol, and it was proved that both the polymers showed an improved efficacy in decreasing the viability of cancer cells and normal cells with the PLGA kaempferol nanoparticle being superior in its specific anticancer activity [70].

Raghavan et al. synthesized gold nanoparticles contain the flavonol, kaempferol, and proved their cell toxic effect on the MCF-7 cancer cell line. The study results proved that the nanoparticles were found to be effective by reducing the vivacity of MCF-7 breast cancer cells as confirmed by the ethidium bromide-acridine orange staining method and urged programmed cell death as confirmed by the CyQuant assay method [71].

\section{Luteolin}

Luteolin, a flavone found in foods which we intake which includes, cabbage, carrots, apple skin, green pepper, and chamomile tea. It was also studies for various therapeutical activities such as antitumorigenic, anti-inflammatory, antidiabetic, and antioxidant [40]. Luteolin requires high bioavailability to exhibit its pharmacological activity in vivo, and due to its low bioavailability in vivo by the oral route, the novel formulation development may prove to be helpful to achieve its maximum pharmacological activity.

Majumdar et al. studied the effect of the bioflavonoid luteolin in nanoparticle formulation to improve its aqueous solubility, bioavailability, and anticancer efficacy of the flavonoid. PLA-PEG-OMe polymer was used for formulating luteolin nanoparticles and the evaluation of the formulated nanoparticles for lung, neck, and head anticancer activity was done by in vitro and in vivo methods. The various cell lines such as H292 and Tu212 were used for the study which showed the inhibitory activity of the nanoparticles against the cancer cell lines and the in vivo anticancer activity by tumor xenograft mouse model which also proved a significant inhibitory effect on the tumor cells [72].

\section{Hesperetin}

Hesperidin, a flavone glycoside, an abundant plant constituent present in limes and lemons, was studied for many pharmacological effects, such as antidiabetic, antioxidant, anticancer, anti-inflammatory effects, and so on. It was found to prevent the cell death. Although it possesses many pharmacological activities, its use in the field of medicine is limited due to its limited aqueous solubility and bioavailability, which can be rectified to a certain extent by formulating the flavonoid as nanoformulation which can also serve as a measure to target the flavonoid to cancer cells.

Gurushankar et al. formulated, characterized, and evaluated the bioflavonoid hesperetin nanoparticles produced using the polymers Eudragit E 100 by the nanoprecipitation technique for its efficiency in treating oral cancer. The results concluded that the nanoparticles of the flavonoid were efficient in producing the toxic effect on the cancer cells on a dose-dependent manner, changes in the mitochondrial membrane potential, improved intracellular reactive oxygen species, necrobiotic morphological changes, and also DNA damage, which urges its development as novel particles for the treatment of dreadful diseases [73].

\section{Naringenin}

Naringenin, chemicallyaflavanoneglycoside is (4,5,7-trihydroxyflavanone) found to be a major constituent in various citrus fruits [74], tomatoes [75], cherries [76], and cocoa is found to be potent anticancer agent in treating liver and gastric cancer $[77,78]$. It was also studied for its antioxidant, liver protective, anti-inflammatory, cardiovascular, gastrointestinal, and immunity-boosting effects [79-84]. Naringenin was found to increase the therapeutic activity of anticancer drugs like doxorubicin, and the drug seems to possess cardiotoxicity on longterm usage, and naringenin seems to possess low bioavailability and solubility which makes it's clear to make modifications in formulations for increasing its pharmacokinetic parameters and also to make the flavonoid disease specific

Winarti et al. studied the in vitro anticancer activity of the flavonoid naringenin nanoparticles formulated using the chitosan polymer. T47D breast cancer cell lines were used for the study and were found that the encapsulated naringenin showed an increased cell toxic effect, and also it resulted in the increased cellular uptake and caused induction in the programmed cell death [85].

Parashar et al studied the naringenin loaded in polycaprolactone polymer as nanoparticles linked with the hyaluronic acid to increase the target specificity of the flavonoid which is a potential drug candidate in treating the lung cancer. The results revealed that the flavonoid naringenin is a potential candidate for treating the lung cancer as proved by the cell toxicity studies on A549 cells and J774 cells [86].

\section{Rutin}

Rutin, a bioflavonoid found in buckwheat bran, citrus fruits, black tea, clove, rose, rue possesses many of the therapeutic activities including the antioxidant activity [87], cardioprotective activity, antiatherosclerotic [36], anticancer activities [88], and many other useful pharmacological properties. Although it has various pharmacological properties, its exploration in the field of medicine is much reduced due to the low solubility and bioavailability, which can be overcome by making modifications in the formulation using various polymers which are of a degradable type and producing the nanoformulations.

Asfour and Mohsen formulated and studied the effect of the less soluble bioflavonoid rutin as nanospheres using the polymer Eudragit S 100 with various concentration of stabilizer poloxamer 188, to target the cancerous cells in the colon region of the body. They proved the rutin nanospheres efficiency in the treatment of colonic cancer as showed by the two-fold increased activity of the nanoformulation in the cell toxic effect of the bioflavonoid rutin [89]

Ahmad et al. developed a nanoemulsion formulation of the bioflavonoid rutin and evaluated for its anticancer activity on the prostatic cancer cell lines PC-3, proving its efficiency in treating prostatic cancer [90].

\section{CONCLUSION}

Many of the daily foods can be used as drugs. Among them, the flavonoids which find their source in many of the daily foods is proved through many experiments to possess potential therapeutic activities in treating many of the diseases. Cancer is considered to be the disease which is of major concern worldwide. Due to the various side effects in using the conventional system of medicine, modern developments have been in full wing in using these plant-derived constituents, especially the flavonoids for the formulation as novel drug delivery system such as nanoparticles, nanoemulsions, and liposomes which will be a boon to the human society in treating cancer.

\section{REFERENCES}

1. Atanasov AG, Waltenberger B, Pferschy-Wenzig EM, Linder T, Wawrosch C, Uhrin P, et al. Discovery and resupply of pharmacologically active plant-derived natural products: A review. Biotechnol Adv 2015;33:1582-614

2. Gurnani N, Mehta D, Gupta M, Mehta BK. Natural products: Source of potential drugs. Afr J Basic Appl Sci 2014;6:171-86.

3. Pawar HA. Natural products as a source of lead to the design of new drugs. Nat Prod Chem Res 2014;2:156.

4. Leopoldini M, Russo N, Chiodo S, Toscano M. Iron chelation by the powerful antioxidant flavonoid quercetin. J Agric Food Chem 2006;54:6343-51. 
5. Lu J, Papp LV, Fang J, Rodriguez-Nieto S, Zhivotovsky B, Holmgren A, et al. Inhibition of mammalian thioredoxin reductase by some flavonoids: Implications for myricetin and quercetin anticancer activity. Cancer Res 2006;66:4410-8.

6. Bukhari SB, Memon S, Mahroof-Tahir M, Bhanger MI. Synthesis, characterization and antioxidant activity copper-quercetin complex. Spectrochim Acta A Mol Biomol Spectrosc 2009;71:1901-6.

7. Kühnau J. The flavonoids. A class of semi-essential food components: Their role in human nutrition. World Rev Nutr Diet 1976;24:117-91.

8. Kumar S, Pandey AK. Antioxidant, lipo-protective and antibacterial activities of phytoconstituents present in Solanum xanthocarpum root. Int Rev Biophys Chem 2012;3:42-7.

9. Hertog MG, Kromhout D, Aravanis C. Flavonoid intake and long term risk of coronary heart disease and cancer in seven countries study. Arch Int Med 1995;155:381-6.

10. Ferrándiz ML, Nair AG, Alcaraz MJ. Inhibition of sheep platelet arachidonate metabolism by flavonoids from Spanish and Indian medicinal herbs. Pharmazie 1990;45:206-8.

11. Ferrándiz ML, Alcaraz MJ. Anti-inflammatory activity and inhibition of arachidonic acid metabolism by flavonoids. Agents Actions 1991;32:283-8.

12. Laughton MJ, Evans PJ, Moroney MA, Hoult JR, Halliwell B. Inhibition of mammalian 5-lipoxygenase and cyclo-oxygenase by flavonoids and phenolic dietary additives. Relationship to antioxidant activity and to iron ion-reducing ability. Biochem Pharmacol 1991;42:1673-81.

13. Alcaraz MJ, Ferrandiz ML. Modification of arachidonic metabolism by flavonoids. J. Ethnopharmacol 1987;21:209-29.

14. Stefani ED, Boffetta P, Deneo-Pellegrini H, Mendilaharsu M, Carzoglio JC, Ronco A, et al. Dietary antioxidants and lung cancer risk: A case-control study in Uruguay. Nutr Cancer 1999;34:100-10.

15. Fotsis T, Pepper MS, Aktas E, Breit S, Rasku S, Adlercreutz H, et al. Flavonoids, dietary-derived inhibitors of cell proliferation and in vitro angiogenesis. Cancer Res 1997:57:2916-21.

16. Caltagirone S, Rossi C, Poggi A, Ranelletti FO, Natali PG, Brunetti M, et al. Flavonoids apigenin and quercetin inhibit melanoma growth and metastatic potential. Int J Cancer 2000;87:595-600

17. Hegarty VM, May HM, Khaw KT. Tea drinking and bone mineral density in older women. Am J Clin Nutr 2000;71:1003-7.

18. Di Carlo G, Autore G, Izzo AA, Maiolino P, Mascolo N, Viola P, et al. Inhibition of intestinal motility and secretion by flavonoids in mice and rats: Structure-activity relationships. J Pharm Pharmacol 1993;45:1054-9.

19. Wang HK, Xia Y, Yang ZY, Natschke SL, Lee KH. Recent advances in the discovery and development of flavonoids and their analogues as antitumor and anti-HIV agents. Adv Exp Med Biol 1998;439:191-225.

20. Wild C, Fasel J. Effect of a flavonoid on the capillary resistance of the rectal mucosa in hepatic cirrhosis. Am J Proctol 1969;20:60-3.

21. Tencate JW, Van Haeringen NJ, Gerritsen J, Glasius E. Biological activity of a semisynthetic flavonoid, O-(o-hydroxy ethyl) rutosine: Light scattering and metabolic studies of human red cells and platelets. Clin Chem 1973;19:31-5.

22. Narayana KR, Reddy MS, Chaluvadi MR, Krishna DR. Bioflavonoids classification, pharmacological, biochemical effects and therapeutic potential. Ind J Pharm 2001;33:2-16.

23. Rawson NE, Ho CT, Li S. Efficacious anti-cancer property of flavonoids from citrus peels. Food Sci Hum Wellness 2014;3:104-9.

24. Kanavos P. The rising burden of cancer in the developing world. Ann Oncol 2006;17 Suppl 8:15-viii23.

25. Devi KP, Balan DJ. Flavonoids and their anti-cancer effect targeting the major signalling pathways involved. In: Recent Advances in the Molecular Mechanism of Flavonoids. Houston, Texas: Studium Press (India Pvt. Ltd.); 2018. p. 1-22.

26. Fernado J, Jones R. The principles of cancer by chemotherapy. Surgery (Oxford) 2015;33:131-5

27. Ravishankar D, Rajora AK, Greco F, Osborn HM. Flavonoids as prospective compounds for anti-cancer therapy. Int J Biochem Cell Biol 2013;45:2821-31

28. Li S, Pan MH, Lai CS, Lo CY, Dushenkov S, Ho CT. Isolation and syntheses of poly-methoxyflavones and hydroxylated polymethoxyflavones as inhibitors ofHL-60 cell lines. Bioorg Med Chem 2007;15:3381-9.

29. Kawaii S, Tomono Y, Katase E, Ogawa K, Yano M. Antiproliferative activity of flavonoids on several cancer cell lines. Biosci Biotechnol Biochem 1999;63:896-9.

30. Saewan N, Koysomboon S, Chantrapromma K. Anti-tyrosinase and anti-cancer activities of flavonoids from Blumea balsamifera DC. J Med Plants Res 2011;5:1018-25.
31. Zhou JR, Mukherjee P, Gugger ET, Tanaka T, Blackburn GL, Clinton SK, et al. Inhibition of murine bladder tumorigenesis by soy isoflavones via alterations in the cell cycle, apoptosis, and angiogenesis. Cancer Res 1998;58:5231-8.

32. Veeramuthu D, Raja WR, Al-Dhabi WA, Savarimuthu I. Flavonoids: Anticancer properties. In: Flavonoids - From Biosynthesis to Human Health. 2017. p. 287-303.

33. Bhagwat RR, Vaidhya IS. Novel drug delivery systems: An overview. Int J Pharm Sci Res 2013;4:970-82

34. Chahar MK, Sharma N, Dobhal MP, Joshi YC. Flavonoids: A versatile source of anticancer drugs. Pharmacogn Rev 2011;5:1-2.

35. Saravanan D, Maheswaran A, Padmavathy J, Angel P. Molecular mechanism of flavonoids on cardiac diseases. In: Devi PK, ediotrs. Recent Advances in the Molecular Mechanism of Flavonoids. Houston, Texas: Studium Press (India Pvt. Ltd.); 2018. p. 183-201.

36. Saha C, Kaushik A, Das A, Pal S, Majumder D. Anthracycline drugs on modified surface of quercetin-loaded polymer nanoparticles: A Dual drug delivery model for cancer treatment. PLoS One 2016;11:e0155710.

37. Devendiran RM, Chinnaiyan SK, Yadav NK, Ramanathan G, Singaravelu S, Perumal SP, et al. Facile synthesis and evaluation of quercetin reduced and dextran sulphate stabilized gold nanoparticles decorated with folic acid for active targeting against breast cancer. RSC Adv 2016:4:1-14.

38. Pandey SK, Patel DK, Thakur R, Mishra DP, Maiti P, Haldar C, et al. Anti-cancer evaluation of quercetin embedded PLA nanoparticles synthesized by emulsified nanoprecipitation. Int J Biol Macromol 2015;75:521-9.

39. Sahu S, Saraf S, Kaur CD, Saraf S. Biocompatible nanoparticles for sustained topical delivery of anticancer phytoconstituent quercetin. Pak J Biol Sci 2013;16:601-9.

40. Shivsharan B, Baburao D, Chandakavathe N. Anti-diabetic potential of Flavonoids and their molecular mechanisms. In: Devi KP, editor. Recent Advances in the Molecular Mechanism of Flavonoids. Houston, Texas: Studium Press (India Pvt.Ltd.); 2018. p. 104-39.

41. Naeimi AF, Alizadeh M. Antioxidant properties of the flavonoid fisetin: An updated review of in vivo and in vitro studies. Trends Food Sci Tech 2017;70:34-44.

42. Wang L, Tu YC, Lian TW, Hung JT, Yen JH, Wu MJ, et al. Distinctive antioxidant and antiinflammatory effects of flavonols. J Agric Food Chem 2006;54:9798-804.

43. Hendriks JJ, de Vries HE, van der Pol SM, van den Berg TK, van Tol EA, Dijkstra CD, et al. Flavonoids inhibit myelin phagocytosis by macrophages; a structure-activity relationship study. Biochem Pharmacol 2003;65:877-85.

44. Inouye $\mathrm{Y}$, Yamaguchi $\mathrm{K}$, Take $\mathrm{Y}$, Nakamura S. Inhibition of avian myeloblastosis virus reverse transcriptase by flavones and isoflavones. J Antibiot (Tokyo) 1989;42:1523-5

45. Chu SC, Hsieh YS, Lin JY. Evaluation of anti- herpetic activity of methanol seed extract and fractions of Securigera securidaca in vitro. J Nat Prod 2013:55:179-83.

46. Lall RK, Adhami VM, Mukhtar H. Dietary flavonoid fisetin for cancer prevention and treatment. Mol Nutr Food Res 2016;60:1396-405.

47. Pal HC, Pearlman RL, Afaq F. Fisetin and its role in chronic diseases. Adv Exp Med Biol 2016;928:213-44

48. Zhang WB, Wang Z, Shu F, Jin YH, Liu HY, Wang QJ, et al. Activation of AMP-activated protein kinase by temozolomide contributes to apoptosis in glioblastoma cells via p53 activation and mTORC1 inhibition. J Biol Chem 2010;285:40461-71.

49. Kim HJ, Kim SK, Kim BS, Lee SH, Park YS, Park BK, et al. Apoptotic effect of quercetin on HT-29 colon cancer cells via the AMPK signaling pathway. J Agric Food Chem 2010;58:8643-50.

50. Kim DY, Park MW, Yuan HD, Lee HJ, Kim SH, Chung SH, et al. Compound $\mathrm{K}$ induces apoptosis via CAMK-IV/AMPK pathways in HT-29 colon cancer cells. J Agric Food Chem 2009;57:10573-8.

51. Ragelle H, Crauste-Manciet S, Seguin J, Brossard D, Scherman D, Arnaud P, et al. Nanoemulsion formulation of fisetin improves bioavailability and antitumour activity in mice. Int $\mathrm{J}$ Pharm 2012;427:452-9.

52. Bothiraja C, Yojana BD, Pawar AP, Shaikh KS, Thorat UH. Fisetinloaded nanocochleates: Formulation, characterisation, in vitro anticancer testing, bioavailability and biodistribution study. Expert Opin Drug Deliv 2014;11:17-29.

53. Ghosh P, Singha Roy A, Chaudhury S, Jana SK, Chaudhury K, Dasgupta $\mathrm{S}$, et al. Preparation of albumin based nanoparticles for delivery of fisetin and evaluation of its cytotoxic activity. Int J Biol Macromol 2016;86:408-17.

54. Meghwal M, Sahu CK. Soy isoflavonoids as nutraceutical for human 
health: An update. J Cell Sci Ther 2015;6:1000194.

55. Suthar AC, Banavalikar MM, Biyani MK. Pharmacological activities of genistein, an isoflavone from soy (Glycine max): Part II - anticholesterol activity, effects on osteoporosis \&amp; menopausal symptoms. Indian J Exp Biol 2001;39:520-5.

56. Zhang H, Liu G, Zeng X, Wu Y, Yang C, Mei L, et al. Fabrication of genistein-loaded biodegradable TPGS-b-PCL nanoparticles for improved therapeutic effects in cervical cancer cells. Int J Nanomedicine 2015;10:2461-73.

57. Phan V, Walters J, Brownlow B, Elbayoumi T. Enhanced cytotoxicity of optimized liposomal genistein via specific induction of apoptosis in breast, ovarian and prostate carcinomas. J Drug Target 2013;21:1001-11.

58. Patel NV, Sheth NR, Mohddesi B. Formulation and evaluation of genistein-Anovel isoflavone loaded chitosan and eudragit $\mathbb{R}$ nanoparticles for cancer therapy. Mat Today: Proceedings 2015;2:4477- 82.

59. Cai L, Yu R, Hao X, Ding X. Folate receptor-targeted bioflavonoid genistein-loaded chitosan nanoparticles for enhanced anticancer effect in cervical cancers. Nanoscale Res Lett 2017;12:509.

60. Aditya NP, Shim M, Lee I, Lee Y, Im MH, Ko S, et al. Curcumin and genistein coloaded nanostructured lipid carriers: In vitro digestion and antiprostate cancer activity. J Agric Food Chem 2013;61:1878-83.

61. Kumar SK, Manju V. Pharmacological applications of isrhamnetin: A short review. Int J Trend Sci Res Dev 2017;1:672-8.

62. Ma G, Yang C, Qu Y, Wei H, Zhang T, Zhang N, et al. The flavonoid component isorhamnetin in vitro inhibits proliferation and induces apoptosis in eca-109 cells. Chem Biol Interact 2007;167:153-60.

63. Lee K, Kwon M, Baek I, Kim H, Lee H, Park H, et al. Ant proliferation effects of isorhamnetin isolated from Persicaria thunbergii on cancer cell lines. Nat Prod Sci 2006;12:214-26.

64. Steffen Y, Gruber C, Schewe T, Sies H. Mono-O-methylated flavanols and other flavonoids as inhibitors of endothelial NADPH oxidase. Arch Biochem Biophys 2008;469:209-19.

65. Settu K, Vaiyapuri M. Formulation and evaluation of isorhamnetin loaded poly lactic-co-glycolic acid nanoparticles. Asian J Pharm Clin Res 2017;10:177-81.

66. Yang RY, Lin S, Kuo G. Content and distribution of flavonoids among 91 edible plant species. Asia Pac J Clin Nutr 2008;17 Suppl 1:275-9.

67. Franke AA, Custer LJ, Arakaki C, Murphy SP. Vitamin C and flavonoid levels of fruits and vegetables consumed in Hawaii. Food Compost Anal 2004;17:1-35.

68. Andrade Cetto A, Wiedenfeld H, Revilla MC, Sergio IA. Hypoglycemic effect of equisetum myriochaetum aerial parts on streptozotocin diabetic rats. J Ethnopharmacol 2000;72:129-33.

69. Calderón-Montaño JM, Burgos-Morón E, Pérez-Guerrero C, LópezLázaro M. A review on the dietary flavonoid kaempferol. Mini Rev Med Chem 2011;11:298-344

70. Luo H, Jiang B, Li B, Li Z, Jiang BH, Chen YC, et al. Kaempferol nanoparticles achieve strong and selective inhibition of ovarian cancer cell viability. Int J Nanomedicine 2012;7:3951-9.

71. Raghavan BS, Kondath S, Anantanarayanan R, Rajaram R. Kaempferol mediated synthesis of gold nanoparticles and their cytotoxic effects on MCF-7 cancer cell line. Process Biochem 2015;50:1966-76.

72. Majumdar D, Jung KH, Zhang H, Nannapaneni S, Wang X, Amin AR, et al. Luteolin nanoparticle in chemoprevention: In vitro and in vivo anticancer activity. Cancer Prev Res (Phila) 2014;7:65-73.

73. Gurushankar K, Gohulkumar M, Prasad NR, Krishnakumar N.
Synthesis, characterization and in vitro anti-cancer evaluation of hesperetin-loaded nanoparticles in human oral carcinoma $(\mathrm{KB})$ cells. Adv Nat Sci Nanosci Nanotechnol 2014;5:15006.

74. Dembinski A, Warzecha Z, Konturek SJ, Ceranowicz P, Dembinski M, Pawlik WW, et al. Extract of grapefruit-seed reduces acute pancreatitis induced by ischemia/reperfusion in rats: Possible implication of tissue antioxidants. J Physiol Pharmacol 2004;55:811-21.

75. Le Gall G, DuPont MS, Mellon FA, Davis AL, Collins GJ, Verhoeyen ME, et al. Characterization and content of flavonoid glycosides in genetically modified tomato (Lycopersicon esculentum) fruits. J Agric Food Chem 2003;51:2438-46.

76. Wang H, Nair MG, Strasburg GM, Booren AM, Gray JI. Antioxidant polyphenols from tart cherries (Prunus cerasus). J Agric Food Chem 1999;47:840-4.

77. Chang H, Mi M, Ling W, Zhu J, Zhang Q, Wei N, et al. Structurally related cytotoxic effects of flavonoids on human cancer cells in vitro. Arch Pharm Res 2008;31:1137-44.

78. Kanno S, Tomizawa A, Hiura T, Osanai Y, Shouji A, Ujibe M, et al. Inhibitory effects of naringenin on tumor growth in human cancer cell lines and sarcoma S-180-implanted mice. Biol Pharm Bull 2005;28:527- 30.

79. Kim JH, Lee JK. Naringenin enhances NK cell lysis activity by increasing the expression of NKG2D ligands on burkitt's lymphoma cells. Arch Pharm Res 2015;38:2042-8.

80. Lee YS, Reidenberg MM. A method for measuring naringenin in biological fluids and its disposition from grapefruit juice by man. Pharmacology 1998;56:314-7.

81. Middleton E Jr., Kandaswami C. Effects of flavonoids on immune and inflammatory cell functions. Biochem Pharmacol 1992;43:1167-79.

82. Rajadurai M, Prince PS. Naringin ameliorates mitochondrial lipid peroxides, antioxidants and lipids in isoproterenol-induced myocardial infarction in wistar rats. Phytother Res 2009;23:358-62.

83. Rimm EB, Katan MB, Ascherio A, Stampfer MJ, Willett WC. Relation between intake of flavonoids and risk for coronary heart disease in male health professionals. Ann Intern Med 1996;125:384-9.

84. Al-Rejaie SS, Abuohashish HM, Al-Enazi MM, Al-Assaf AH, Parmar MY, Ahmed MM, et al. Protective effect of naringenin on acetic acid-induced ulcerative colitis in rats. World J Gastroenterol 2013;19:5633-44.

85. Winarti L, Sari LO, Nugroho AE. Naringenin loaded chitosan nanoparticles formulation and it's in vitro evaluation against T47D breast cancer cell line. Indones J Pharm 2015;26:147-57.

86. Parashar P, Rathor M, Dwivedi M, Saraf SA. Hyaluronic acid decorated naringenin nanoparticles: Appraisal of chemopreventive and curative potential for lung cancer. Pharmaceutics 2018;10:E33.

87. Kamalakkannan N, Prince PS. Antihyperglycaemic and antioxidant effect of rutin, a polyphenolic flavonoid, in streptozotocin-induced diabetic wistar rats. Basic Clin Pharmacol Toxicol 2006;98:97-103.

88. Luo H, Jiang BH, King SM, Chen YC. Inhibition of cell growth and VEGF expression in ovarian cancer cells by flavonoids. Nutr Cancer 2008;60:800-9.

89. Asfour MH, Mohsen AM. Formulation and evaluation of $\mathrm{pH}$-sensitive rutin nanospheres against colon carcinoma using HCT-116 cell line. J Adv Res 2018;9:17-26.

90. Ahmad M, Sahabjada AS, Akhtar J, Hussain A, Badaruddeen M, Arshad M, et al. Development of a new rutin nanoemulsion and its application on prostate carcinoma PC3 cell line. EXCLI J 2017; 16:810-23. 